\title{
The Role of Casein Kinase 2 in Ion Channel Remodeling After Myocardial Infarction
}

\author{
Editorial to: "Valsartan Upregulates Kir2.1 in Rats Suffering from Myocardial Infarction \\ via Casein Kinase 2" by Xinran Li et al.
}

Seungwoo Kang ${ }^{1}$

Published online: 25 July 2015

(C) Springer Science+Business Media New York 2015

Cardiac remodeling is a necessary process in the context of physiological adaptation during normal growth according to the demand of alterations, including changes in contractility and beating rate. However, malfunctional remodeling due to pathological conditions is an ultimate disaster in disease pathogenesis, which leads to a poor outcome and high mortality [1]. Besides the cardiac enlargement of ventricular volume after myocardial infarction (MI), dysfunction due to electrophysiological remodeling such as a decrease in delayed rectifier potassium currents (IK) is a prominent feature showing reduced survival in patients with heart disease [2]. Cardiac inward rectifying potassium currents (Ik1) play an important role in shaping action potentials, which is a key character representing the electrical activity made by a multitude of various ion channels and transporters [3]. The cardiac IK1 stabilizes the resting membrane potential and is responsible for shaping the initial depolarization and final repolarization of the action potential $[4,5]$. Indeed, infarction generally entails significant cellular and molecular remodeling in the left ventricle, resulting in functional and biochemical alterations of the myocardium due to the modulation of IK1 [2].

Therefore, focusing on a strategy to attenuate the pathogenic remodeling, such as decrease in IK1 after MI has been receiving increasing attention. Notably, a delayed rectifier potassium channel, Kir2.1, which is encoded by the KCNJ2 gene, has been studied because it has been demonstrated that

Seungwoo Kang

seungwoo.kang@rutgers.edu

1 Department of Anesthesiology, Pharmacology and Physiology, Rutgers, The State University of New Jersey, New Jersey Medical School, 185 South Orange Avenue, Newark, New Jersey 07103, USA
Kir2.1 expression is decreased after MI [6]. Also, overexpression of Kir2.1 channel in embryonic stem cell-derived cardiomyocytes attenuates post-transplantation proarrhythmic risk in myocardial infarction [7]. Previously, studies have shown the mechanisms undergoing transcriptional modulation of Kir2.1 after myocardial infarction. For example, upregulation of microRNA-1 (miR-1) and consequent reduction of Kir2.1 is observed in rat ventricular myocytes in a rodent model of MI. This is due to the increase in serum response factor (SFR), a transcriptional activator of the miR-1 gene [8].

In the current issue of Cardiovascular Drugs and Therapy, $\mathrm{Li}$ et al. report evidence for kinase-dependent modulation of IK1 in an MI rodent model [9]. This work not only identifies a new potential target to attenuate the risk of heart failure after MI, but also provides additional information on the mechanisms of how valsartan, an angiotensin II type I receptor blocker attenuates the effect of MI. First, they induce the MI in male Wistar rats by ligation of the coronary artery, which upregulates casein kinase 2 (CK2) expression and downregulates Kir2.1 expression in myocytes in the left ventricle. In addition, in rat ventricular cells, CK2 overexpression leads to reduction of Kir2.1 expression. This effect is abolished by 4,5,6,7-Tetrabromobenzotriazole (TBB), a cellpermeable selective inhibitor of CK2, treatment. Also, this biochemical data is confirmed by electrophysiological data showing IK1 current density in cultured neonatal rat ventricular cardiomyocytes is decreased by CK2 overexpression and this effect is abolished by TBB treatment. Using an Electrophoretic mobility shift assay (EMSA), the regulation of the CK2 activity after MI was confirmed.

Most of all, this provides the new insight that $\mathrm{CK} 2$ has an important role in MI-induced ion channel remodeling. Although it has been demonstrated that CK2 has an important role in modulating cardiac hypertrophy; for example, CK2 induces development of hypertrophic phenotypes in rat 
neonatal cardiomyocytes via activation of HDAC2 by its phosphorylation at the S394 residue [10], most research studying the role of CK2 in cardiac pathophysiology have focused on cardiac hypertrophy but have rarely focused on ion channel remodeling. In this issue, $\mathrm{Li}$ et al. demonstrate that in left ventricular myocytes after MI, there is kinase-derived modulation of transcription of ion channels. Second, in a series of biochemical experiments, $\mathrm{Li}$ and colleagues show the effect of valsarten on the CK2 and Kir2.1 expression in the rodent MI model. Valsartan has been widely used for patients suffering from hypertension. Indeed, valsartan inhibits the reninangiotensin system by blocking the angiotensin II type 1 receptor (AT1), which prevents the increase in blood pressure induced by circulating angiotensin II resulting in potent antihypertensive activity $[11,12]$. In the current article, Li et al. conclude that valsartan ameliorates IK1 remodeling after MI in the rodent MI model by attenuating CK2-induced decrease in Kir2.1 channel expression. Thus, this provides additional mechanisms of how valsartan attenuates the effect of MI via modulation of ion channel remodeling.

Consistent with other studies, this work also raises the following questions. First, in terms of the physiological balance in the posttranslational process, kinases have a counterpart to feedback its role [13]. Are there candidates of protein phosphatase as a counterpart in CK2's role in MI-derived ion channel remodeling like Phosphatase and tensin homolog (PTEN) or Protein phosphatase 1 (PP1)? If CK2 is the candidate to modulate Kir2.1 expression following MI, what can be the counterpart of the kinase working in this situation? Second, $\mathrm{Li}$ et al. provide the role of CK2 to modulate Kir2.1 expression via activation of a transcriptional process. Are there any other direct effects of CK2 to reduce the capacity of the Kir2.1 channel via direct phosphorylation of the channel, leading to consequential internalization of the ion channel into the cytoplasm? Since the Kir2.1 channels have 8 CK2 phosphorylation site motifs [14] and CK2 activation is increased after MI in the rodent model, it would be interesting to look at the direct effect of CK2 in the Kir2.1 channel remodeling after MI. It will be also helpful to identify other combinational or synergistic effects by CK2 such as depletion of Phosphatidylinositol 4,5-bisphosphate (PIP2) in MI-induced ion channel remodeling.

CK2 has been considered to be a regulator of over 300 substrates [15]. These new findings raise further questions and the studies into its potential therapeutic application are still in the early experimental stage. However, Kir2.1 remains an exciting target for improving heart function after MI and in terms of the perspective of potential therapeutics, whether CK2 can modulate Kir2.1 expression after MI, leading to a change of IK1 is important information. For the treatment after MI, this could be a potential target to develop a strong additional layer of protection. It is certain that developing approaches to target the ion channel remodeling system will assist in improving heart function to prevent heart failure after MI and other heart-related disorders.

\section{References}

1. Sutton MG, Sharpe N. Left ventricular remodeling after myocardial infarction: pathophysiology and therapy. Circulation. 2000;101: 2981-8.

2. Kaprielian R, Sah R, Nguyen T, Wickenden AD, Backx PH. Myocardial infarction in rat eliminates regional heterogeneity of AP profiles, $\mathrm{I}(\mathrm{to}) \mathrm{K}(+)$ currents, and $[\mathrm{Ca}(2+)](\mathrm{i})$ transients. Am J Physiol Heart Circ Physiol. 2002;283:H1157-68.

3. Carmeliet E. Cardiac ionic currents and acute ischemia: from channels to arrhythmias. Physiol Rev. 1999;79:917-1017.

4. Chilton L, Ohya S, Freed D, George E, Drobic V, Shibukawa Y, et al. $\mathrm{K}+$ currents regulate the resting membrane potential, proliferation, and contractile responses in ventricular fibroblasts and myofibroblasts. Am J Physiol Heart Circ Physiol. 2005;288: H2931-9.

5. Diaz RJ, Zobel C, Cho HC, Batthish M, Hinek A, Backx PH, et al. Selective inhibition of inward rectifier $\mathrm{K}+$ channels (Kir2.1 or Kir2.2) abolishes protection by ischemic preconditioning in rabbit ventricular cardiomyocytes. Circ Res. 2004;95:325-32.

6. Li X, Chu W, Liu J, Xue X, Lu Y, Shan H, et al. Antiarrhythmic properties of long-term treatment with matrine in arrhythmic rat induced by coronary ligation. Biol Pharm Bull. 2009;32:1521-6.

7. Liao SY, Tse HF, Chan YC, Mei-Chu Yip P, Zhang Y, Liu Y, et al. Overexpression of Kir2.1 channel in embryonic stem cell-derived cardiomyocytes attenuates posttransplantation proarrhythmic risk in myocardial infarction. Heart Rhythm. 2013;10:273-82.

8. Shan H, Li X, Pan Z, Zhang L, Cai B, Zhang Y, et al. Tanshinone IIA protects against sudden cardiac death induced by lethal arrhythmias via repression of microRNA-1. Br J Pharmacol. 2009;158: 1227-35.

9. Li X, Hu H, Wang Y, et al. Valsartan upregulates Kir2.1 in rats suffering from myocardial infarction via casein Kinase 2. Cardiovasc Drugs Ther 2015;29:this issue

10. Eom GH, Cho YK, Ko JH, Shin S, Choe N, Kim Y, et al. Casein kinase-2alphal induces hypertrophic response by phosphorylation of histone deacetylase $2 \mathrm{~S} 394$ and its activation in the heart. Circulation. 2011;123:2392-403.

11. Yilmaz EN, Vahl AC, van Rij GL, Vink GQ, Brom HL, Rauwerda $J A$. The effect of inhibition of renin-angiotensin system by valsartan during hypovolemic shock and low flow sigmoideal ischaemia in pigs. Cardiovasc Surg. 2003;11:45-51.

12. Bissessor N, White H. Valsartan in the treatment of heart failure or left ventricular dysfunction after myocardial infarction. Vasc Health Risk Manag. 2007;3:425-30.

13. Kang S, Xu M, Cooper EC, Hoshi N. Channel-anchored protein kinase $\mathrm{CK} 2$ and protein phosphatase 1 reciprocally regulate KCNQ2-containing M-channels via phosphorylation of calmodulin. J Biol Chem. 2014;289:11536-44.

14. Correia MJ, Wood TG, Prusak D, Weng T, Rennie KJ, Wang HQ. Molecular characterization of an inward rectifier channel (IKir) found in avian vestibular hair cells: cloning and expression of pKir2.1. Physiol Genomics. 2004;19:155-69.

15. Litchfield DW. Protein kinase CK2: structure, regulation and role in cellular decisions of life and death. Biochem J. 2003;369:1-15. 